\title{
Dynamical weakening of pyroclastic flows by mechanical vibrations
}

\author{
Jose Manuel Valverde ${ }^{1, \star}$, Carlos Soria-Hoyo ${ }^{1}$, and Olivier Roche ${ }^{2}$ \\ ${ }^{1}$ Faculty of Physics. University of Seville. Ada. Reina Mercedes s/n 41012 Seville, Spain \\ ${ }^{2}$ Laboratoire Magmas et Volcans. Université Blaise Pascal-CNRS-IRD, OPGC. 6 Avenue Blaise Pascal, TSA 60026-CS 60026, \\ F-63178 Aubière, France
}

\begin{abstract}
Dynamical weakening of dense granular flows plays a critical role on diverse geological events such as seismic faulting and landslides. A common feature of these processes is the development of fluidsolid relative flows that could lead to fluidization by hydrodynamic viscous stresses. Volcanic ash landslides (pyroclastic flows) are characterized by their high mobility often attributed to fluidization of the usually fine and/or low-density particles by their interaction with the entrapped gas. However, the physical mechanism that might drive sustained fluidization of these dense granular flows over extraordinarily long runout distances is elusive. The behavior of volcanic ash in a slowly rotating drum subjected to mechanical vibrations shown in this work suggests that fluid-particle relative oscillations in dense granular flows present in volcanic eruption events can promote pore gas pressure at reduced shear rates as to sustain fluidization.
\end{abstract}

Pyroclastic flows, triggered by the collapse of lava domes and explosive volcanic eruptions, represent a serious hazard for densely populated areas around volcanoes. These dense granular flows are chiefly characterized by their high mobility. Usually, extraordinary long runout distances over $100 \mathrm{~km}$ and across topographic obstacles are reached [2-5]. A possible mechanism responsible for such astonishing behavior is sustained fluidization caused by the enhancement of pore gas pressure [2,5]. Since pyroclastic flows commonly contain large amounts of fine ash and light pumice rock fragments, their flow dynamics could be indeed determined by the prevalence of the interstitial gas drag force on the particles over their weight. The role of fluidization on the extraordinary mobility of pyroclastic flows is suggested by the typical signature observed in the patterns of prehistoric ignimbrite deposits indicating negligible friction and suppressed turbulence at the depositional boundary layer [3]. It is believed that long runout landslides involving large release and deposit volumes $\left(>10^{6} \mathrm{~m}^{3}\right)$ are associated with high flow velocities ranging from tens to hundreds of $\mathrm{m} / \mathrm{s}$ [4]. At such high velocities granular flows of fine and/or light particles become fluidized by the entrapped gas at the avalanche front and acquire the behavior of a low viscosity fluid lacking any resistance to shear [6]. Recent observations of sediments from dense pre-historic pyroclastic flows showing the signatures of fluidization and traveling distances over $170 \mathrm{~km}$ demonstrate relatively modest and uniform velocities $(<10$ $\mathrm{m} / \mathrm{s}$ ) suggesting that high velocities are not necessary for fluidization of pyroclastic flows [5]. These observations, together with lab-scale experiments, indicate that the main factor in driving sustained fluidization is long-lived gas

\footnotetext{
^e-mail: jmillan@us.es
}

pore pressure presumably due to hampered diffusion by the reduced permeability of the trapped gas through large amounts of fine ash [5].

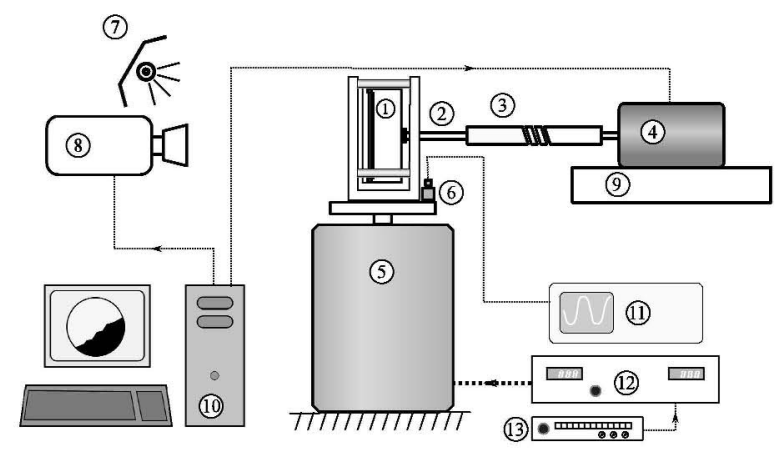

Figure 1. Experimental setup: (1) rotating drum (side view), (2) aluminium shaft, (3) flexible coupling (cardan), (4) step motor and controller, (5) vibrator (Tira TV 52120), (6) accelerometer, (7) halogen lamp, (8) CCD camera, (9) optical antivibration table, (10) desktop computer, (11) oscilloscope, (12) power amplifier, (13) function generator.

In our work we investigate the possible role of mechanical vibrations on sustained fluidization of pyroclastic flows at reduced flow velocities by means of lab-scale experiments. In a previous work [7] vibrations were shown to significantly weaken the dynamical resistance of a cornstarch fine powder and glass beads to avalanche in a slowly rotating drum. In the present paper, experimental measurements are shown using ignimbrite powder samples from prehistoric pyroclastic flows deposits. Long-lived high intensity mechanical vibrations could be generated in pyro- 
clastic flows by collisions of pyroclasts onto the mountain slope or other obstacles $[8,9]$ with a seismic energy that can be comparable with the energy released by the precursory swarm of volcano-tectonic earthquakes [10]. These intense vibrations could play a role on the enhancement of pore fluid pressure as suggested, for example, by the correlation found between the volume of pyroclastic flows during the Unzen volcano eruption (1991) and the magnitude of the generated earthquakes [10].

We have used ignimbrite powder samples from pyroclastic deposits at Clermont-Ferrand area (France), which consisted of mainly three types of particles: i) pumices as the most abundant, white, vesiculated, with typical density of $0.5-2 \mathrm{~g} / \mathrm{cm}^{3}$ that represent the fragmented magma; ii) rock fragments commonly dark, with typical density of $2.5-3 \mathrm{~g} / \mathrm{cm}^{3}$ coming from the conduit or the ground; and, by a small amount, iii) crystals of several colors mainly in size range $\sim 250 \mu \mathrm{m}-1 \mathrm{~mm}$. The average solid density of the samples measured by a gas pycnometer was $\rho_{p}=2.4 \mathrm{~g} / \mathrm{cm}^{3}$. As a common feature regarding their morphology, Scanning Electron Microscopy pictures show a marked angular shape of the particles with a noticeable presence of sub-micron fragments and asperities on their surface. Since the gas-solid hydrodynamic interaction is strongly dependent on particle size the powder as received was sieved at $100^{\circ} \mathrm{C}$ to avoid ambient moisture induced cohesion in order to obtain samples in diverse ranges of particle size $d_{p}$ (between $<63 \mu \mathrm{m}$ and $2 \mathrm{~mm}$ ).

Figure 1 shows a layout of the experimental setup used in our work. The powder half-fills a cylindrical Plexiglas drum ( $4.5 \mathrm{~cm}$ internal radius and $2 \mathrm{~cm}$ depth), which is mounted on the base of an electromagnetic vibration exciter and slowly rotated $(\Omega \simeq 4.8 \mathrm{rpm}$ ) around its horizontal axis. The vibrator is driven by a signal generator that provides sinusoidal, vertical vibrations of controlled amplitude $A$, and frequency $f$ (in the range $25-200 \mathrm{~Hz}$ ). The shaft is fitted at the other end to the motor axis by means of an elastic cardan to isolate the motor from vibrations. A CCD camera interfaced to a computer for image processing records the profile of the powder as affected by rotation and vibration. In order to avoid moisture induced cohesion the samples were heated up to $100^{\circ} \mathrm{C}$ immediately before the test, which serves also to mimic the high temperature usually found in pyroclastic flows. Peak vibration velocity $u_{1}=A 2 \pi f$ is monitored using a piezoelectric accelerometer and image analysis.

Figure 2 illustrates the effect of adding vibrations to the slowly rotating drum. As may be seen vibrations have a relevant influence on the dynamical behavior of the ignimbrite samples regardless of their particle size. The slowly rotated bed loses progressively its frictional strength as the intensity of vibrations is increased. For sufficiently strong vibrations, the angle of the slope drops to nearly zero even for the largest particle size samples. A remarkable behavior is observed for the finest ignimbrite sample $\left(d_{p}<63 \mu \mathrm{m}\right)$ that resembles the behavior of relatively large glass beads $\left(d_{p}>1 \mathrm{~mm}\right)$ fluidized by liquids [11]. Instabilities in these liquid-fluidized beds are characterized by the early appearance of a succession of es-

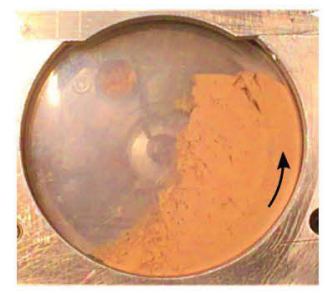

a)
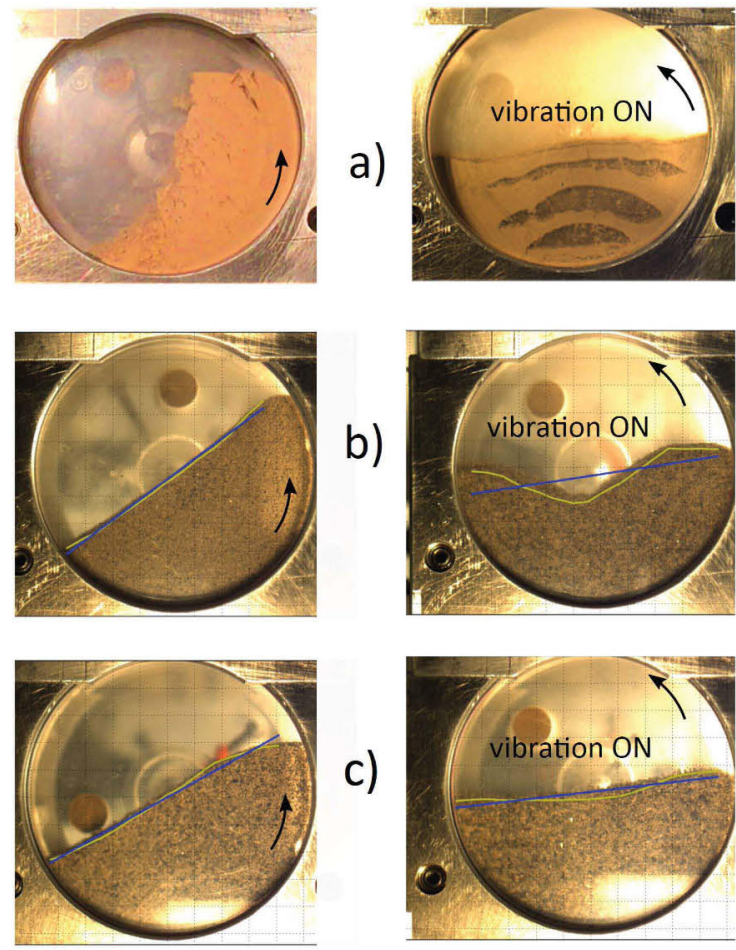

C)
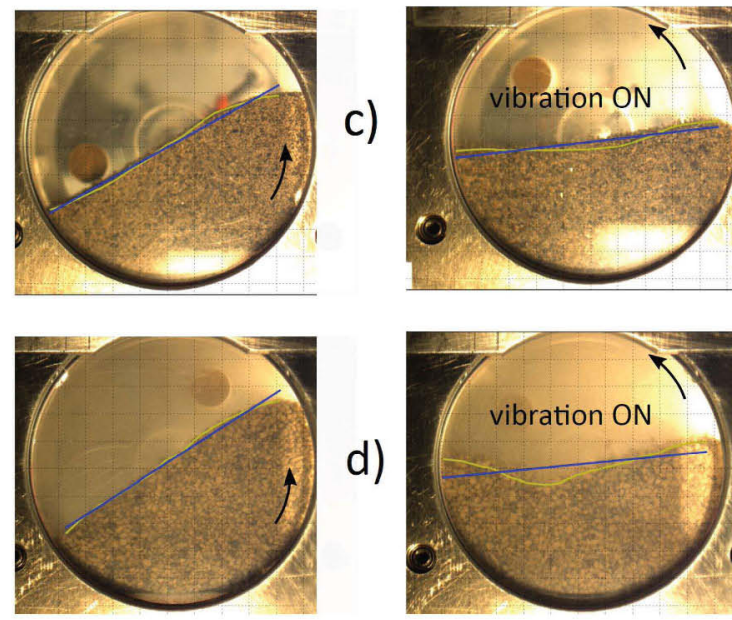

d)

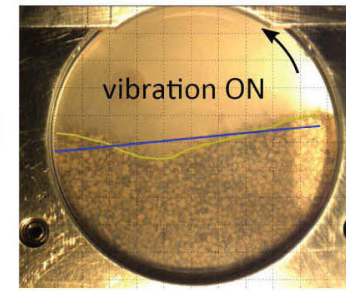

Figure 2. Snapshots of the slowly rotating drum half-filled with ignimbrite powder samples in the absence of vibrations (left) and for high intensity vibrations $(f=200 \mathrm{~Hz}$, vibration velocity $0.1 \mathrm{~m} / \mathrm{s}$ ) applied (right). a) $d_{p}<63 \mu \mathrm{m}$; b) $250-500 \mu \mathrm{m}$; c) 500 $\mu \mathrm{m}-1 \mathrm{~mm}$;) $1-2 \mathrm{~mm}$.

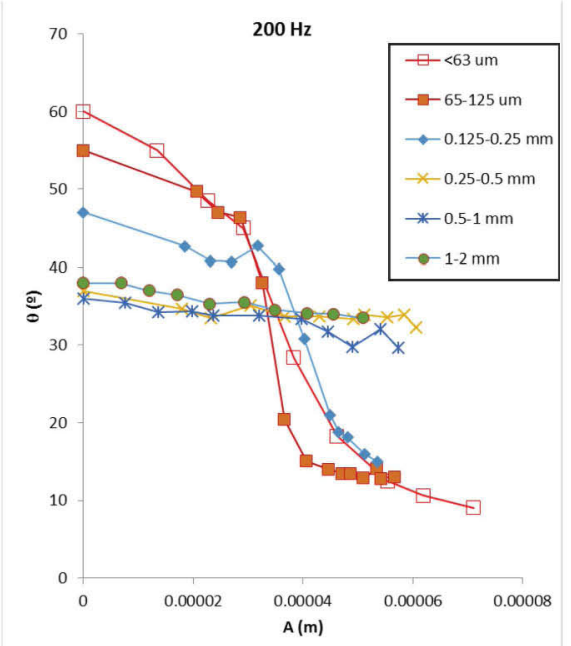

Figure 3. Average angle of avalanche in the slowly rotating drum as a function of the amplitude of vertical vibrations imposed $(f=$ $200 \mathrm{~Hz}$ ) for ignimbrite samples of diverse particle size ranges as indicated. 
sentially one-dimensional upward traveling voidage wavefronts (1-DTWs) whose destabilization at sufficiently high liquid flow rates lead to bubble-like voidage pockets [11]. A similar flow structure evolution is observed in our rotating drum experiment for the finest ignimbrite sample as the vibration intensity is increased indicating the extraordinary relevance of gas-solid hydrodynamic interactions as promoted by vibrations. Figure $2 \mathrm{a}$ demonstrates the formation of these gas pockets arising for destabilization of 1-DTWs in close analogy with observations in liquidfluidized beds. For the larger particle size $\left(d_{p}>100 \mu \mathrm{m}\right)$ ignimbrite samples, the flow regime in the rotating drum becomes inertial in the absence of vibrations since the gasparticle drag force is negligible as compared to inertial forces [6]. Yet, we see that the addition of vibrations leads also to a loss of resistance to shear (Figures 2b-d). A characteristic feature observed in some of the experiments is the formation of a double slope (Fig. 2b) arguably caused by boundary effects due to the small diameter of the drum used in our tests. This undesirable effect would be avoided by using drums of much larger diameter, which was prevented in our setup due to technical limitations related to lack of mechanical stability by the simultaneous imposition of rotation and vibrations but should be a subject for future work.

Figure 3 shows data on the average angle of avalanche as a function of the vibration amplitude $(f=200 \mathrm{~Hz})$. The effect of vibration becomes more relevant as particle size is decreased which indicates a marked enhancement of the gas-solid hydrodynamic interaction for the small particle size samples. Thus, it may be foreseen that the effect of mechanical vibrations on the weakening of pyroclastic flows would be promoted as the amount of fine particles is increased. As seen in Fig. 3, a sudden drop of the angle occurs in the range of vibration amplitudes around $A=0.03-0.05 \mathrm{~mm}$ for samples with particle size below $0.25 \mathrm{~mm}$, which would be caused by the transition to fluidization of most of the material.

According to a simple model on the fluid pore pressure due to relative gas-solid oscillations in a granular medium (see [7] for a detailed description), the relative decrease of the friction coefficient in the avalanching powder would be given by

$$
\frac{\Delta \mu}{\mu}=\beta \Upsilon u_{1}^{\prime}
$$

where $\beta=\frac{6 \pi \eta R \Lambda n_{0}}{\rho_{p} \phi g}, \Lambda \simeq \frac{E \phi}{18(1-\phi)^{3}}, \Upsilon=$ $\left[\left(1+\frac{R}{\delta}\right)^{2}+\left(\frac{R}{\delta}\right)^{2}\left(1+\frac{2 R}{9 \delta}\right)^{2}\right]^{1 / 2}, \quad \eta$ is the fluid dynamic viscosity, $R$ is the particle radius (spherical particles are assumed), $\phi$ is the particle volume fraction, $E \simeq 180$ is the Ergun's constant, $\delta=\sqrt{\frac{\eta}{\rho \omega}}$ is the thickness of the Stokes boundary layer, $\rho$ is the fluid density, $\omega=2 \pi f$, and $u_{1}^{\prime}$ is the r.m.s oscillation velocity $\left(u_{1}^{\prime}=u_{1} / \sqrt{2}\right)$. Data on the relative decrease of the friction coefficient (absolute value) estimated from the average angle of avalanche in our rotating drum experiments are plotted in Fig. 4 as a function of $\beta \Upsilon u_{1}^{\prime}$. To calculate $\beta \Upsilon$ average values of particle size were taken as $d_{p}=63 \mu \mathrm{m}, 95 \mu \mathrm{m}, 188$ $\mu \mathrm{m}, 325 \mu \mathrm{m}, 750 \mu \mathrm{m}$, and $1.5 \mathrm{~mm}$, respectively. The particle volume fraction for each sample in the drum was calculated from the volume filled and the solid density. In the case of the finest ignimbrite, the particle volume fraction is small ( $\phi \simeq 0.25$ ) as typical for fine cohesive powders [6]. As particle size is increase, $\phi$ increases up to $\phi \simeq 0.4$ for the noncohesive samples. This is still a low value as compared with the packing fraction of randomly packed noncohesive hard spheres $(\phi \simeq 0.56)$. The reason why ignimbrite particles pack in more porous structures is their irregular shape [12]. Ignimbrite particles are notably angular shaped, which affects also both the interparticle attractive force and the gas-solid hydrodynamic drag. The latter would be decreased as the contact surface area is reduced whereas the former would be increased as the surface to volume ratio is increased for low sphericity particles. This can been accounted introducing the sphericity $\Psi$ of the particles in the effective Ergun's constant $(E / \Psi)$ [13]. Sphericity of volcanic ash particles typically varies in the range $\Psi \simeq 0.6-0.8$ [14]. In our calculations we have used $\Psi=0.7$.

As seen in Fig. 4 experimental data on the relative decrease of the friction coefficient follows the trend predicted despite the model simplicity albeit the predicted result deviates considerably from data for the lowest frequencies tested and the smallest particle size powders. In these limits the relative decrease of the static friction coefficient is fairly below the theoretical expectation. On the other hand, Fig. 4 shows a good agreement between the experimental data obtained for the relative decrease of the friction coefficient and the prediction by Eq. 1 for the largest particle size powders suggesting that the enhancement of pore pressure by vibrations weakens the inertial flow of these noncohesive granular materials that otherwise (under steady flows) would not be affected by the gas-solid hydrodynamic interaction [6]. Probable causes for the deviation observed in the case of fine powders may be compaction induced vibration and the prevalence of van der Waals attractive forces between the particles on their weight, which confers the powder a nonnegligible cohesiveness although the typical angular shape of the particles as well as the large presence of asperities on their surface would lead to a reduction of van der Waals forces [15]. A consequence of the relative strength of interparticle attractive forces is that the angle of avalanche depends critically on the typical size of the system [16]. Moreover, interparticle attractive forces are usually enhanced by consolidation forces [17], which would increase the frictional resistance of the powder. Adhesive forces lead also to the aggregation of particles of size typically below $\sim 50 \mu \mathrm{m}$ due to their prevalence over particle weight [18]. These aggregates screen external fluid flow and may behave as low density effective grains that can be fluidized at reduced velocities and exhibit a liquid-like behavior [18].

Dynamical weakening by oscillatory flows can be expected in a variety of geological process involving intense vibrations and/or acoustic waves of very long duration and high frequencies leading to viscous fluid-solid oscillatory 

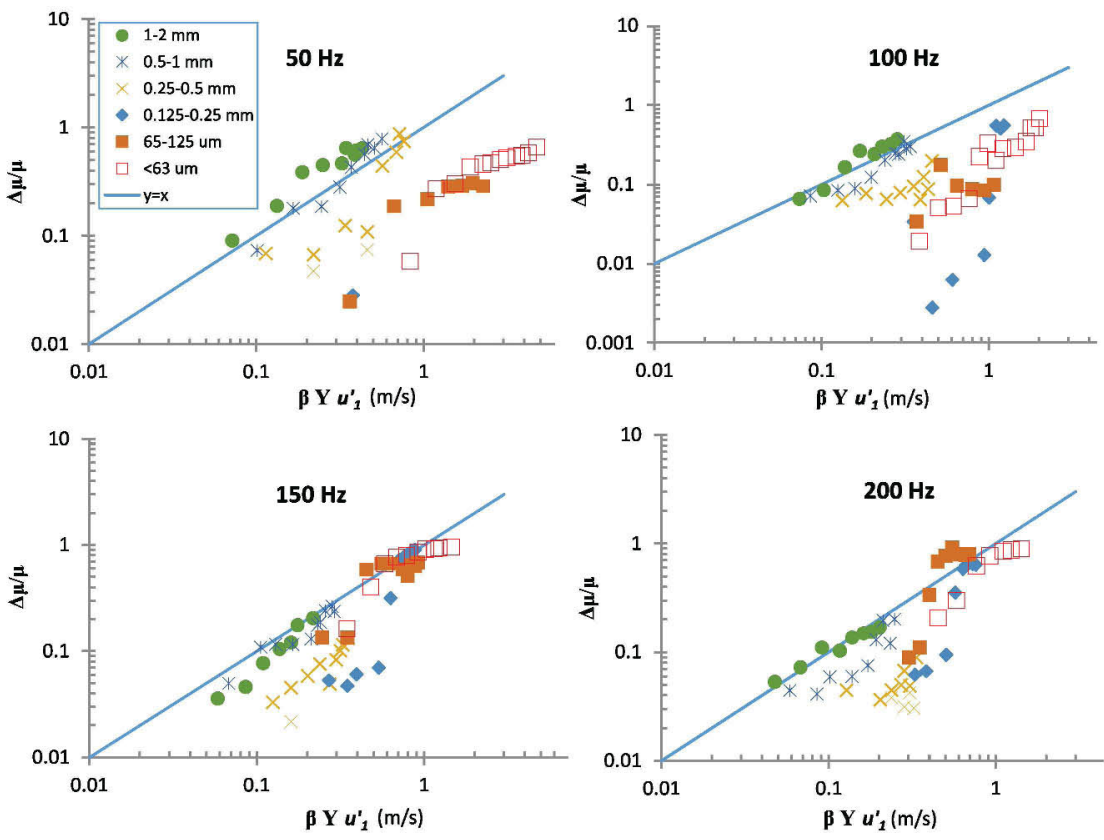

Figure 4. Relative decrease (absolute value) of the friction coefficient measured for ignimbrite samples of various particle size ranges in a slowly rotating drum subjected to vibrations of diverse frequencies as a function of $\beta \Upsilon u_{1}^{\prime}$ (Eq. 1).

flows. This may be the case of seismic faulting, lahars, non-volcanic landslides, detachment faulting and impact crater formation. A major outstanding question for understanding the flow behavior of this variety of geological processes is what is the physical mechanism that drives dynamical weakening as a commonly observed feature in all of them. Our results suggest that the enhancement of pore fluid pressure by viscous oscillatory flows could be a strong mechanism to be given further consideration.

\section{Acknowledgements}

This work was supported by the Spanish Government Agency Ministerio de Economia y Competitividad and FEDER Funds (Contract CTQ2014-52763-C2-2-R).

\section{References}

[1] O. Roche, M.A. Gilbertson, J.C. Phillips, R.S.J. Sparks, Journal of Geophysical Research: Solid Earth 109, 1 (2004), b10201

[2] R. Cas, H. Wright, C. Folkes, C. Lesti, M. Porreca, G. Giordano, J. Viramonte, Bulletin of Volcanology 73, 1583 (2011)

[3] O. Korup, D. Schneider, C. Huggel, A. Dufresne, in Treatise on Geomorphology, edited by J.F. Shroder (Academic Press, San Diego, 2013), pp. 183 - 199, ISBN 978-0-08-088522-3

[4] O. Roche, D.C. Buesch, G.A. Valentine, Nature Communications 7, 10890 (2016)
[5] A. Castellanos, J.M. Valverde, A.T. Perez, A. Ramos, P.K. Watson, Phys. Rev. Lett. 82, 1156 (1999)

[6] J.M. Valverde, C. Soria-Hoyo, Journal of Geophysical Research: Solid Earth 120, 6182 (2015), 2015JB012317

[7] K. Uhira, H. Yamasato, M. Takeo, Journal of Geophysical Research: Solid Earth 99, 17757 (1994)

[8] S. De Angelis, V. Bass, V. Hards, G. Ryan, Natural Hazards and Earth System Science 7, 467 (2007)

[9] V.M. Zobin, in Introduction to Volcanic Seismology (Second Edition), edited by V.M. Zobin (Elsevier, Oxford, 2012), pp. 261 - 293, second edition edn., ISBN 978-0-444-56375-0

[10] S. Sundaresan, Annu. Rev. Fluid Mech. 35, 63 (2003)

[11] R.Y. Yang, R.P. Zou, A.B. Yu, Phys. Rev. E 62, 3900 (2000)

[12] P. Carman, Chemical Engineering Research and Design 75, Supplement, S32 (1997)

[13] C.M. Riley, W.I. Rose, G.J.S. Bluth, Journal of Geophysical Research: Solid Earth 108, n/a (2003), 2504

[14] A. Castellanos, Adv. Phys. 54, 263 (2005)

[15] J.M. Valverde, A. Castellanos, A. Ramos, P.K. Watson, Phys. Rev. E 62, 6851 (2000)

[16] M.A.S. Quintanilla, A. Castellanos, J.M. Valverde, Phys. Rev. E 64, 031301(1) (2001)

[17] A. Castellanos, J.M. Valverde, M.A.S. Quintanilla, Phys. Rev. Lett. 94, 075501(1) (2005) 